\title{
Review of: "Motion of water monomers reveals a kinetic barrier to ice nucleation on graphene"
}

\author{
Changqing Sun ${ }^{1}$ \\ 1 Nanyang Technological University
}

Potential competing interests: The author(s) declared that no potential competing interests exist. No competing interests

I have been working in the field of Water, Ice, and Aqueous Solutions since 2010 and have extended the understanding of water ice from the perspective of molecular motion scheme to the premise of hydrogen bond ( $\mathrm{O}: \mathrm{H}-\mathrm{O}$ with ":" being the electron lone pair) cooperativity with the $\mathrm{O}-\mathrm{O}$ repulsion as the key to coupling the inter- and intramolecular interactions [1, 2]. As a coupling oscillator pair, the O:H-O cooperativity in its segmental length, energy, vibration frequency, and the specific heat dictates the extraordinary adaptivity, cooperativity, recoverability, and sensitivity of water and ice [3]. I would like to share my humble views on the sophisticated work reported in [4].

The work is interesting and important, particularly, discoveries on the ice nucleation of separated molecules, at 100-130 K temperatures, on hydrophobic substrate. Using helium spin-echo (HeSE) spectroscopy, the team revealed that water molecular dipoles at sufficiently large separation preferentially adsorb on either hollow or bridge sites [5] of the graphene/Ni(001) in the same polar direction pointing $\mathrm{H}-\mathrm{O}$ downward to the substrate. At longer times (time $>600 \mathrm{~s}$ ) and higher coverage the molecules reorient to adopt the hydrogen bonded configuration. This work is sophisticatedly planned and executed, which contributes significantly to the understanding of ice formation at extremely low temperatures, on hydrophobic substrate, with evolution of the molecular distance.

From my personal experience and observations, I would like to recommend the following possible consequences and mechanisms for further discussion and exploration:

1. The p-electronic field of graphene defines the orientation of largely-separated molecules. The electrification also shortens and stiffens the $\mathrm{H}-\mathrm{O}$ bond and polarizes the molecules. The O:H distance should be longer and softer than they are in the bulk [6].

2. As molecular coverage increases, the rule of ice configuration becomes dominance, which overcomes the p-electronic polarization, forming the tetrahedrally-structured ice without proton translational tunnelling taking place because of the high $\mathrm{H}-\mathrm{O}$ energy of $4.0 \mathrm{eV}$ [7].

3. The structural transition takes place at 100-130 K within the quasisolid regime. Molecular undercoordination and electrification depress the temperature $T_{N}$ for ice nucleation substantially [8]. 
4. Electrification or molecular undercoordination shortens and stiffens the $\mathrm{H}-\mathrm{O}$ bond while the $\mathrm{O}: \mathrm{H}$ nonbond responds to the perturbation contrastingly. The $\mathrm{H}-\mathrm{O}$ cohesive energy governs the melting temperature $T_{m}$ whereas the O:H energy dictates the $T_{N}$ through the superposition of the segmental specific-heat curves. Thus, supercooling happens to water ice under electrification by salt solvation and molecular undercoordination by nanodroplet formation, for instances [9-12].

Critiques and discussions are welcome and appreciated.

1. Sun, C.Q. and Y. Sun, The Attribute of Water: Single Notion, Multiple Myths. Vol. 131. 2016: Springer-Verlag. 525.

2. Sun, C.Q., Solvation Dynamics: A Notion of Charge Injection. 2019: Springer Nature. 326.

3. Huang, Y.L., et al., Hydrogen-bond relaxation dynamics: Resolving mysteries of water ice. Coord Chem Rev, 2015. 285: p. 109-165.

4. Tamtögl, A., et al., Motion of water monomers reveals a kinetic barrier to ice nucleation on graphene. Nature Communications, 2021. 12(1): p. 3120.

5. Böttcher, S., et al., Graphene on ferromagnetic surfaces and its functionalization with water and ammonia. Nanoscale research letters, 2011. 6(1): p. 1-7.

6. Sun, C.Q., Water electrification: Principles and applications. Advances in Colloid and Interface Science, 2020. 282: p. 102188.

7. Huang, Y., et al., Size, separation, structure order, and mass density of molecules packing in water and ice. Scientific Reports, 2013. 3: p. 3005.

8. Sun, C.Q., et al., Density, Elasticity, and Stability Anomalies of Water Molecules with Fewer than Four Neighbors. Journal of Physical Chemistry Letters, 2013. 4: p. 2565-2570.

9. Lide, D.R., CRC Handbook of chemistry and physics, 80th Ed. 1999, Boca Raton: CRC Press

10. Sun, C.Q., et al., Density and phonon-stiffness anomalies of water and ice in the full temperature range. Journal of Physical Chemistry Letters, 2013. 4: p. 3238-3244.

11. Mallamace, F., et al., The anomalous behavior of the density of water in the range $30 \mathrm{~K}<T<373$ K. Proceedings of the National Academy of Sciences of the United States of America, 2007. 104(47): $p$. 18387-91.

12. Erko, M., et al., Density minimum of confined water at low temperatures: a combined study by small-angle scattering of X-rays and neutrons. PCCP, 2012. 14(11): p. 3852-8. 